\title{
Exact Hydrodynamic Description of Active Lattice Gases
}

\author{
Mourtaza Kourbane-Houssene, ${ }^{1}$ Clément Erignoux, ${ }^{2}$ Thierry Bodineau,${ }^{3}$ and Julien Tailleur ${ }^{1}$ \\ ${ }^{1}$ Université Paris Diderot, Sorbonne Paris Cité, MSC, UMR 7057 CNRS, 75205 Paris, France \\ ${ }^{2}$ Instituto de Matemática Pura e Aplicada, Rio de Janeiro, Brazil \\ ${ }^{3}$ CMAP, Ecole polytechnique, CNRS, Université Paris-Saclay, 91128, Palaiseau, France
}

(Dated: January 30, 2018)

\begin{abstract}
We introduce a class of lattice gas models of active matter systems whose hydrodynamic description can be derived exactly. We illustrate our approach by considering two systems exhibiting two of the most studied collective behaviours in active matter: the motility-induced phase separation and the transition to collective motion. In both cases, we derive coupled partial differential equation describing the dynamics of the local density and polarization fields and show how they quantitatively predict the emerging properties of the macroscopic lattice gases.
\end{abstract}

Active matter systems are intrinsically out of thermal equilibrium due to the dissipation of energy at the microscopic scales to produce motion [1]. The resulting non-Brownian random walks endow these systems with a rich phenomenology, from the long-range order observed in 2D assemblies of self-propelled particles [58] to the spatio-temporal chaos of dense assemblies of nematic particles [9, 10] through the enhanced clustering resulting from the interplay of repulsive forces and self-propulsion [11 13].

The toolbox of equilibrium statistical mechanics cannot be used a priori to describe such non-thermal systems and one has to rely on dynamical studies, even to characterize systems in a steady state. When an effective detailed-balance with respect to a non-Boltzman distribution is (partially) restored [14-16], this can only be established after a complex, case-by-case study of otherwise analytically untractable dynamics. Numerical simulations have thus become a prominent tool to study active matter, and progress is often hindered by strong finitesize effects [17. In such contexts, exact results derived on simple model systems can offer much needed guiding principles. Whereas this has frequently been true outside equilibrium, for instance to characterize dynamical phase transitions [18], little success has been achieved along these lines for active matter systems. In particular, the derivation of coarse-grained descriptions of active systems has attracted a lot of interest over the past decades [19 31, but the complexity of the underlying microscopic models has prevented the derivation of exact results outside the mean-field regime [32, 33].

In this letter, following the recent interest for lattice models of active particles [27, 31, 34, 39, we introduce a new class of active lattice gas models whose large-scale physics can be described exactly. We build on recent developments in the mathematical-physics literature to derive exact hydrodynamic descriptions of lattice gases [4043. For illustration purposes, we focus on two of the most studied emergent behaviours in active systems: the motility-induced phase separation (MIPS) [11, 15, 44] and the transition to collective motion [5, 17, 45], but the approach we present here can be extended beyond these cases. For both systems, we single out the relevant hydrodynamic modes and construct their exact dynamics. This allows us both to simulate efficiently their large scale behaviours as well as analytically study their instabilities and the corresponding phase diagrams.

We first consider a microscopic lattice gas which exhibit MIPS. $N$ particles evolve on a discrete ring of $\alpha L$ sites. There are two types of particles and each site is occupied by at most one particle so that a configuration can be represented using occupation numbers $\sigma_{i}$ at site $i$ with values in $\{-1,0,1\}$. To model self-propulsion, we endow the + particles with a weak drift to the right and the - particles with a weak drift to the left, in addition to a symmetric diffusive motion. Furthermore, a particle can tumble and change sign at fixed rate. More precisely, the dynamics combine 3 mechanisms:

1.1 For each bond $(i, i+1), \sigma_{i}$ and $\sigma_{i+1}$ are exchanged at rate $\mathrm{D}$.

1.2 For each bond $(i, i+1)$, a + particle in $i$ jumps to $i+1$ if $\sigma_{i+1}=0$ or a - particle in $i+1$ jumps to $i$ if $\sigma_{i}=0$, with rate $\lambda / L$.

1.3 Particles switch sign at rate $\gamma / L^{2}$.

The total number of particles is $N \equiv \rho_{0} \alpha L$ where $\rho_{0} \in$ $[0,1]$ stands for the mean density. The system remains homogeneous for small $\rho_{0}$ or $\lambda$, whereas the homogeneous phases become unstable for large densities and drift. The previous dynamics can be generalized to higher dimensions. We show in Fig. 1 the result of $2 \mathrm{D}$ numerical simulations leading to the coexistence between dilute and dense phases typical of MIPS. Depending on whether the $2 \mathrm{D}$ case is built solely with a left-right bias or whether one considers biases along each of the four directions, we observe different symmetries for the coexistence phases.

To account for this phenomenology, one needs to characterize the evolution of the local density of particles. The expectation of the microscopic variables can be computed from the dynamical rules. For example, let 

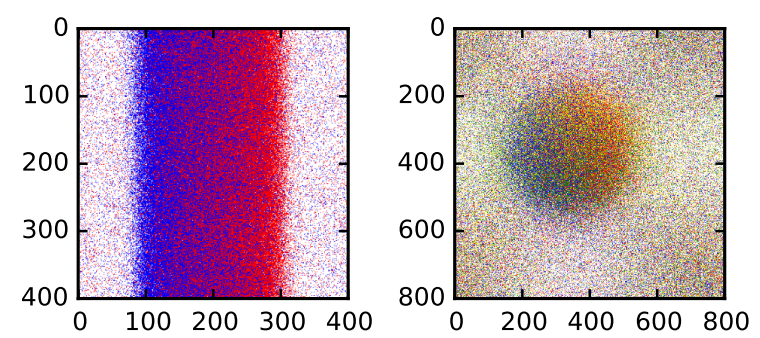

Figure 1. Snapshots of microscopic simulations of dynamics 1.1-1.3 in 2D showing MIPS for lattices of $\alpha L \times \alpha L$ sites, with $L=100$. The symmetry of the dense phase depends whether particles are biased only along $\hat{x}$ (left, $\alpha=4, \rho_{0}=0.65$ ) or can point along the four lattice bonds (right, $\alpha=8, \rho_{0}=0.63$ ). The color of a site gives the direction of a particle: blue for $\rightarrow$, green for $\uparrow$, red for $\leftarrow$, yellow for $\downarrow$. Simulations parameters: $D=1, \gamma=10, \lambda=40$.

$$
\begin{aligned}
\sigma_{i}^{ \pm}(t) & =1_{\left\{\sigma_{i}(t)= \pm 1\right\}} \text { then } \\
\partial_{t}\left\langle\sigma_{i}^{+}\right\rangle & =D\left[\left\langle\sigma_{i+1}^{+}\right\rangle+\left\langle\sigma_{i-1}^{+}\right\rangle-2\left\langle\sigma_{i}^{+}\right\rangle\right]-\frac{\gamma}{L^{2}}\left[\left\langle\sigma_{i}^{+}\right\rangle-\left\langle\sigma_{i}^{-}\right\rangle\right] \\
& +\frac{\lambda}{L}\left[\left\langle\sigma_{i-1}^{+}\left(1-\left|\sigma_{i}\right|\right)\right\rangle-\left\langle\sigma_{i}^{+}\left(1-\left|\sigma_{i+1}\right|\right)\right\rangle\right]
\end{aligned}
$$

These equations are however not closed, since the evolution of $\left\langle\sigma_{i}^{+}(t)\right\rangle$ involves the correlator $\left\langle\sigma_{i}^{+}(t)\left|\sigma_{i+1}(t)\right|\right\rangle$.

A closed, explicit description of the dynamics can, however, be achieved at the macroscopic level. Indeed, following 40 43], we chose the three processes above to occur with rates scaling with $L$ in such a way that they all contribute equally to a hydrodynamic regime obtained by a diffusive rescaling of space and time: $x=i / L$ and $\tau=t / L^{2}$. Indeed, the first dynamical rule leads to the diffusion of the particles: if one follows the particles without their signs, the exchange dynamics 1.1 amount to a symmetric simple exclusion process (SSEP). This first rule makes particles travel a distance $\Delta i \sim L$ on a time $\delta t \sim L^{2}$ and hence at a macroscopic scale $\Delta x \sim 1$ for $\Delta \tau \sim 1$. The second rule applies at a reduced rate $\lambda / L$, but provides a systematic drift to the left or to the right depending on the particle type. Similarly, in a time $L^{2}$, this leads to a displacement of order $L$. Finally at an even more reduced rate $\gamma / L^{2}$, the particle type changes which boils down to saying that a particle changes direction once in a macroscopic unit of time. This occurs sufficiently rarely so that the drift has a macroscopic effect between two updates.

To derive the hydrodynamic description of the system in the large $L$ limit, we introduce the macroscopic densities as

$$
\rho^{ \pm}(x, \tau) \simeq \frac{1}{2 L^{\delta}} \sum_{|i-L x| \leqslant L^{\delta}} 1_{\left\{\sigma_{i}\left(\tau L^{2}\right)= \pm 1\right\}},
$$

where the coarse graining scale is determined by the parameter $\delta \in(0,1)$. Note that the microscopic system size $\alpha L$ depends on two parameters: $1 / L$ plays the
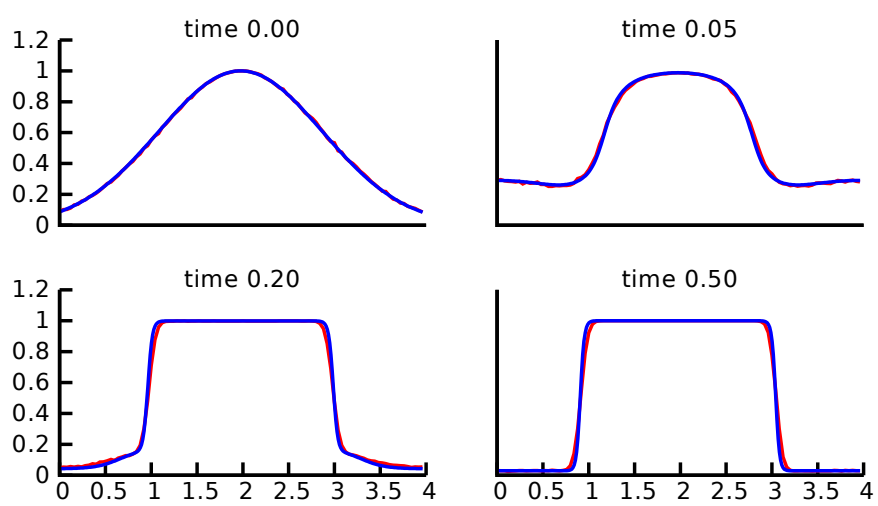

Figure 2. Successive snapshots leading to phase coexistence in 1D: the microscopic (red) and macroscopic (blue) simulations agree quantitatively. Simulation parameters: $D=1$, $\lambda=5, \gamma=0.1, \rho_{0}=0.75, \alpha=4$. Microscopic simulations: Continuous time simulation of dynamics 1.1-1.3 with $L=1000$. The density profiles of the microscopic simulations are averaged over 200 independent runs; Macroscopic simulations: Semi-spectral method with $n=50$ modes and a semi-implicit Euler time-stepping with $d t=10^{-4}$.

role of a microscopic mesh; it vanishes in the $L \rightarrow \infty$ limit in which $\alpha$ then controls the rescaled system size $x \in[0, \alpha]$. The macroscopic equations for the densities $\left(\rho^{+}, \rho^{-}\right)$, starting from a smooth initial condition, can then be derived exactly as

$$
\begin{aligned}
& \partial_{\tau} \rho^{+}=D \partial_{x}^{2} \rho^{+}-\lambda \partial_{x}\left[\rho^{+}(1-\rho)\right]-\gamma\left(\rho^{+}-\rho^{-}\right), \\
& \partial_{\tau} \rho^{-}=D \partial_{x}^{2} \rho^{-}+\lambda \partial_{x}\left[\rho^{-}(1-\rho)\right]+\gamma\left(\rho^{+}-\rho^{-}\right),
\end{aligned}
$$

where the total density is $\rho=\rho^{+}+\rho^{-}$. The mathematical method to rigorously derive these hydrodynamic equations has been initiated in [40, 41] and we refer to [43] for a detailed implementation. We will explain below the underlying principles. Intuitively, Eq. (3) can be deduced from the microscopic equation (1) by first replacing the discrete differences by derivatives. Justifying the forms of the non-linear advection terms require to close the twopoint correlations in (1). Even though on a macroscopic scale the three mechanisms of the dynamics compete at equal footing, the first one dominates locally as it occurs much more frequently. Thus, in the large $L$ limit, it can be shown that the local correlations are controlled by the stirring part. The invariant measures of the dynamics reduced to the stirring part are product Bernoulli measures indexed by two parameters which prescribe the local densities of \pm particles. Thus, at any time, the local statistics of the full dynamics are determined by a product of Bernoulli measures parametrized by the local densities (2). The approximation by these local measures is valid beyond the expectation of the correlations and applies at the level of sample paths so that local averages as in (2) converge with high probability to the solution of the hydrodynamic equations (3)-(4). Note that the hydrodynamic equations directly extend to higher dimensions. 
We compare in Fig. 2 simulations of the microscopic and macroscopic dynamics for the $1 \mathrm{D}$ case. A perfect agreement between the two dynamics is observed on their way to phase coexistence.

To analyze the emerging behaviours predicted by (3)(4), we first introduce an unnormalized polarization field $m=\rho^{+}-\rho^{-}$. The dynamics can then be reduced to a dimensionless form using $\rho=\bar{\rho} \tilde{\rho}, m=\bar{\rho} \tilde{m}, x=\ell \tilde{x}$ and $t=\tau \tilde{t}$ where $\bar{\rho}=1, \tau=\frac{1}{\gamma}, \ell=\sqrt{\frac{D}{\gamma}}$, so that $\tilde{x} \in$ $\left[0 ; \alpha \sqrt{\frac{\gamma}{D}}\right]$. In this system of units the evolution equation reads:

$$
\begin{aligned}
\partial_{t} \rho & =\Delta \rho-\operatorname{Pe} \nabla(m(1-\rho)) \\
\partial_{t} m & =\Delta m-\operatorname{Pe} \nabla(\rho(1-\rho))-2 m
\end{aligned}
$$

with $\mathrm{Pe}=\frac{\lambda}{\sqrt{D \gamma}}$ and where, for the sake of clarity, we have omitted the tilde notation (we stick to the rescaled variables until the end of the discussion of this model). Eqs. (5) and (6) show that the system is fully characterized by two control parameters: the density $\rho_{0}=N / L$ and the Péclet number Pe. The latter compares the length traveled between two tumbles thanks to the drift, $\lambda / \gamma$, to the one resulting from the diffusive dynamics, $\sqrt{D / \gamma}$. For small Péclet numbers, the diffusion dominates and the effect of self-propulsion is negligible. Conversely, the effect of activity gets more and more pronounced as Pe increases.

The homogeneous solutions of Eqs. (5)-(6), $\rho(x, t)=$ $\rho_{0}$ and $m(x, t)=0$, are linearly unstable when

$$
\operatorname{Pe}^{2}\left(1-\rho_{0}\right)\left(2 \rho_{0}-1\right)>2 .
$$

For any $\mathrm{Pe}$ larger than a critical value $\mathrm{Pe}^{\mathrm{c}}=4$, the system is thus linearly unstable for $\rho_{0} \in\left[\rho_{l}^{s}, \rho_{h}^{s}\right]$ with $\rho_{l, h}^{s}=\frac{3}{4} \pm \frac{1}{4} \sqrt{1-\frac{16}{\mathrm{Pe}}}$. This defines the spinodal region of the system. Note that this is a large wavelength instability, observed only for macroscopic system size $L_{\alpha} \equiv \alpha \sqrt{\frac{\gamma}{D}}>\frac{2 \pi}{\sqrt{\mathrm{Pe}^{2}\left(1-\rho_{0}\right)\left(2 \rho_{0}-1\right)-2}}$. We now turn to the computation of the coexisting densities, generalizing the method introduced in [46].

For simplicity, we consider the $2 \mathrm{D}$ case with left-right bias in which the interfaces between the phases are flat and along $\hat{y}$. We consider fully phase-separated profiles and use Eqs. (5)-(6) to construct a domain-wall solution describing the evolution of the density and magnetization fields through an interface. In the steady-state, Eq. (5) simply leads to $m=\frac{1}{\mathrm{Pe}} \frac{\nabla \rho}{1-\rho}$. Eq. $[6]$ then reads $\partial_{x} g=0$ with

$$
g \equiv g_{0}(\rho)+\Lambda(\rho)\left(\partial_{x} \rho\right)^{2}-\kappa(\rho) \partial_{x x} \rho
$$

where $\Lambda(\rho)^{-1}=-\operatorname{Pe}(1-\rho)^{2}, \kappa(\rho)^{-1}=\operatorname{Pe}(1-\rho)$, and $g_{0}(\rho)=\operatorname{Pe} \rho(1-\rho)-2 \log (1-\rho) /$ Pe. Since the density is homogeneous in the gas and liquid phases, one gets a first relationship between the coexisting densities:

$$
g_{0}\left(\rho_{g}\right)=g_{0}\left(\rho_{\ell}\right) \equiv \bar{g}(\mathrm{Pe})
$$

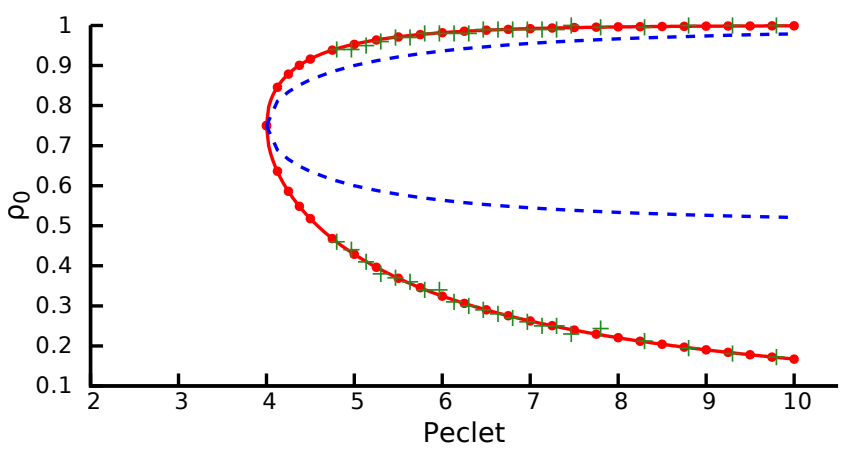

Figure 3. Phase diagram of the MIPS observed for dynamics 1.1-1.3. For each Peclet number, the coexisting phases are computed analytically (red line), by simulating the microscopic process (black \& green dots) and by numerically solving the macroscopic equations (red crosses). The analytic predictions for the spinodals are shown in blue.

We now introduce a function $R(\rho)$ such that $R^{\prime \prime} \kappa=$ $-\left(2 \Lambda+\kappa^{\prime}\right) R^{\prime}$ and $\phi(R)$ such that $\phi^{\prime}(R)=g_{0}(\rho)$. Computing $I=\int_{x_{g}}^{x_{\ell}} g \partial_{x} R d x$ leads to

$$
I=\bar{g}\left[R\left(\rho_{\ell}\right)-R\left(\rho_{g}\right)\right]=\Phi\left(R_{\ell}\right)-\Phi\left(R_{g}\right) .
$$

Eq. 10 then enforces the equality of $h_{0} \equiv \phi^{\prime}(R) R-\phi(R)$ between the two phases. The function $R$ and $\phi$ can be computed explicitly as

$$
R(\rho)=\log (1-\rho) ; \quad \Phi(R)=\operatorname{Pe}\left(1-\frac{e^{R}}{2}\right) e^{R}-\frac{R^{2}}{\mathrm{Pe}}
$$

The binodals can then be computed from the equality of $g_{0}$ and $h_{0}$ between the two phases, which amounts to a common tangent construction on $\phi(R)$. The resulting phase diagram is shown in Fig 3 . It shows perfect agreement with both simulations of the hydrodynamic equations and of the microscopic models in $1 \mathrm{D}$ and 2D. As far as we are aware, this is the first microscopic model for which the hydrodynamic description and the phase diagram of a motility-induced phase separation can be derived exactly.

We now turn to the phase transition leading to collective motion which is probably the most studied emerging behaviour in active matter [1, 5, 8, 17, 27, 45]. Following the strategy laid out in the first part of this letter, we introduce a microscopic model of polar aligning active particles and derive its hydrodynamic limit exactly. For simplicity, we first describe the model in one dimension. $N$ particles evolve on a discrete ring of $\alpha L$ sites. Each particle is described by two degrees of freedom: its position $i \in\{1 \ldots L\}$ and its orientation, noted \pm in 1D. We call $\eta_{i}=\left(\eta_{i}^{+}, \eta_{i}^{-}\right) \in \mathbb{N}^{2}$ the number of particles of each type on site $i$. The dynamics of a \pm particle is given by the three following processes:

2.1 Symmetric hops with rate $2 D$ 

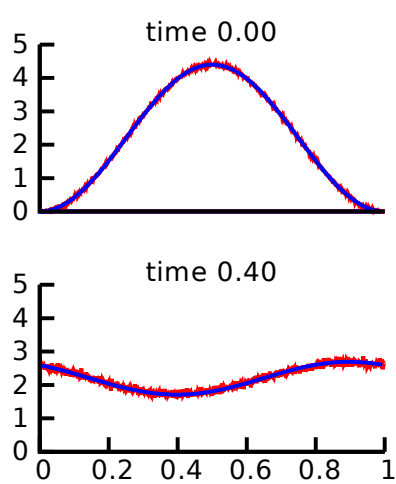

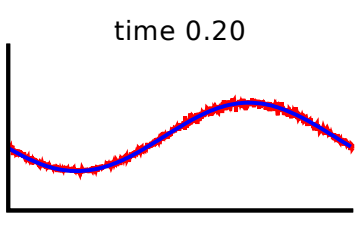

time 1.00

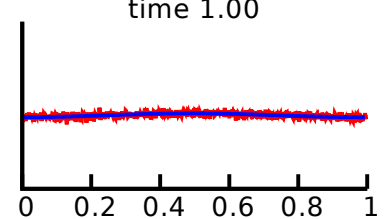

Figure 4. Successive snapshots of one-dimensional simulations of the microscopic dynamics 2.1-2.3 and of the hydrodynamic equations (17) and (18). The system is in the ordered phase and the initial condition is fully ordered with $\rho(x, t)=\rho_{0}[1+\cos (2 \pi x)]$. Microscopic simulations: the density profiles are obtained by averaging over 300 continuous time simulations of the dynamics 2.1-2.3 on a lattice of $L=1000$ sites $(\alpha=1)$; Macroscopic simulations: pseudo-spectral simulations with 50 Fourier modes and a semi-implicit time-stepping with $d t=10^{-4}$. Physical parameters: $D=0.5, \lambda=4, \beta=0.8$.

\subsection{Jumps from site $i$ to $i \pm 1$ with rate $\frac{\lambda}{L}$}

2.3 Flips into a $\mp$ particle with rate $\frac{1}{L^{2}} c_{i}^{ \pm}\left(\eta_{i}^{+}, \eta_{i}^{-}\right)$ where we choose $c^{ \pm}$to produce a polar alignement

$$
c^{ \pm}\left(\eta_{i}^{+}, \eta_{i}^{-}\right)=\exp \left[\mp \beta\left(\eta_{i}^{+}-\eta_{i}^{-}\right)\right]
$$

We consider again a diffusive rescaling of time and space to obtain the exact hydrodynamic equations

$$
\begin{aligned}
& \partial_{t} \rho^{+}=D \Delta \rho^{+}+\lambda \nabla \rho^{+}-F\left(\rho^{+}, \rho^{-}\right) \\
& \partial_{t} \rho^{-}=D \Delta \rho^{-}-\lambda \nabla \rho^{-}+F\left(\rho^{+}, \rho^{-}\right)
\end{aligned}
$$

where the functions $F\left(\rho^{+}, \rho^{-}\right)$are given by

$$
F\left(\rho^{+}, \rho^{-}\right)=f^{+}\left(\rho^{+}, \rho^{-}\right)-f^{-}\left(\rho^{+}, \rho^{-}\right)
$$

and $f^{ \pm}\left(\rho^{+}(x), \rho^{-}(x)\right)$ are the averages of $n^{ \pm} c^{ \pm}\left(n_{i}^{+}, n_{i}^{-}\right)$ with respect to the local Poisson measure

$$
\nu_{\rho^{+}, \rho^{-}}\left(n_{i}^{+}, n_{i}^{-}\right)=\mathrm{e}^{-\rho^{+}-\rho^{-}} \frac{\left(\rho^{+}\right)^{n_{i}^{+}}}{\left(n_{i}^{+}\right) !} \frac{\left(\rho^{-}\right)^{n_{i}^{-}}}{\left(n_{i}^{-}\right) !} .
$$

Again, while the dynamical rules $2.1-2.3$ all contribute equally in the hydrodynamic scaling, the symmetric random walk equilibrates much faster on the microscopic mesh scale. The averages of the non-linear contributions due to the flipping rules are thus computed with respect to local Poisson measures, which are the steady-state measures of the symmetric random walk, conditionned to producing the correct mean local densities of + and particles. Finally, the hydrodynamic equations $130-14$ can be rewritten in the more familiar form

$$
\begin{aligned}
\partial_{t} \rho & =D \Delta \rho+\lambda \nabla m \\
\partial_{t} m & =D \Delta m+\lambda \nabla \rho-2 \tilde{F}(m, \rho)
\end{aligned}
$$

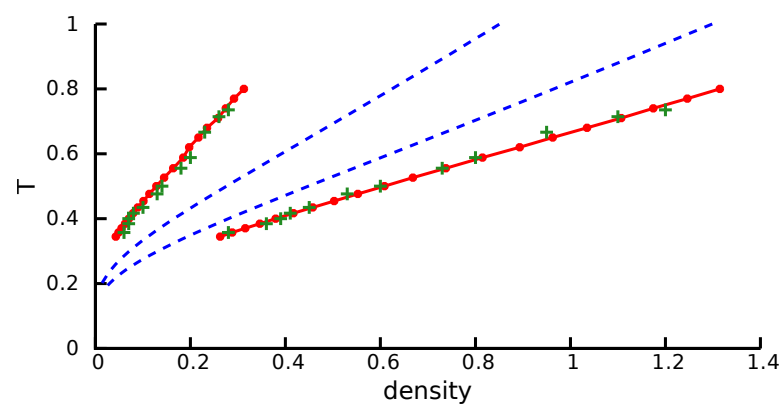

Figure 5. Phase diagram of the flocking transition observed for dynamics 2.1-2.3 in the $T, \rho$ plane for $D=0.5$ and $\lambda=1$. The spinodals are shown in blue. Simulations of the hydrodynamics equations (red lines) and the microscopic model (green dots) lead to coexisting densities which are in agreement within the accuracy of our simulations.

where

$$
\tilde{F}=(m \operatorname{ch}[m \operatorname{sh}(\beta)]-\rho \operatorname{sh}[m \operatorname{sh}(\beta)]) e^{-\beta+\rho \operatorname{ch}(\beta)-\rho}
$$

Note that $\tilde{F}$ is not equal to the mean-field expectation of $n^{+} c^{+}-n^{-} c^{-}$.

Simulations of the microscopic model and its hydrodynamic description confirm the presence of a transition to collective motion. At large 'temperature' $T \equiv \beta^{-1}$ and low density $\rho_{0}=N / V$, the system is in a homogeneous disordered 'gas' phase. At low noise and large density, the system is in a homogeneous ordered liquid phase with a non-zero average flux of particles in the steady state. These homogeneous phases are separated by a coexistence region in a which a dilute disordered gas of density $\rho_{g}(T)$ coexist with a dense liquid phase of density $\rho_{\ell}(T)$. A perfect agreement between simulations of the microscopic model and of its hydrodynamic description is shown in Fig. 4 where the relaxation of a perturbation towards a homogeneous liquid phase is shown.

For this microscopic model, the similarity of this transition to collective motion with a liquid-gas phase transition in the canonical ensemble can now be derived analytically. The hydrodynamic description indeed predicts that the disordered homogeneous phase $\rho(x)=$ $\rho_{0}, m(x)=0$ loses linear stability for densities such that $\partial_{m} \tilde{F}(0, \rho)<0$, i.e. $\rho_{0}>\rho_{s}^{g} \equiv \operatorname{sh}(\beta)^{-1}$. Then, a fully ordered solution appears given by $\rho(x)=\rho_{0}$ and $m_{0}$ the solution of

$$
\frac{m_{0}}{\rho_{0}}-\tanh \left[m_{0} \operatorname{sh}(\beta)\right]=0 .
$$

This solution is however linearly unstable for $\rho_{s}^{g}<\rho_{0}<$ $\rho_{s}^{\ell}$ and leads to the travelling bands described above. As far as we are aware, this is the first time that the existence of a region in which neither homogeneous phases 
is stable is rigorously proven. At higher densities, for $\rho_{0} \geqslant \rho_{s}^{\ell}$, the ordered uniform state becomes linearly stable. In agreement with the liquid-gas scenario proposed for the flocking transition [27, 45], the coexisting densities are such that $\rho_{g}<\rho_{s}^{g}<\rho_{s}^{\ell}<\rho_{\ell}$. The corresponding phase diagram is shown in Figure 5 . The coexisting densities observed for the microscopic model agree with their macroscopic counterparts within the numerical accuracy of our simulations.

In this Letter we have introduced a class of lattice models for which one can derive exact hydrodynamic equations. Our strategy relies on scaling the rates of the different dynamical contributions so that they contribute equally at a diffusive hydrodynamic scale. The symmetric hopping however controls the dynamics at a local, mesoscopic scale. This yields an explicit form for the local measure one needs to use to compute the average of any non-linear function entering the dynamics of the mean local densities. Then, the hydrodynamic descriptions allow us to characterize the large scale emerging behaviours of these active lattice gases. In particular we have introduced two models presenting two of the most studied collective behaviours of active particles, namely the Motility-Induced Phase Separation and the transition to collective motion. Constructing more general models is rather straightforward using the ingredients presented in this letter, for instance to study nematic alignement or the interplay between MIPS and aligning torques. Note that the hydrodynamic description is exact for finite macroscopic times in the $L \rightarrow \infty$ limit. For large-but-finite sizes, describing the statistics of the active lattice-gas trajectories requires the addition of subleading fluctuating terms, in the spirit of the Macroscopic Fluctuation Theory [18, 47, 48, These terms are key to understanding the selection of meta-stable propagating solutions observed in simple flocking models [45, 49, 50]; their rigorous mathematical derivation, however, remains an open challenge.

Acknowledgment We thank Alexandre Solon and Hugues Chaté for interesting discussions. JT and MKH acknowledge support from the ANR grant Bactterns. CE and TB acknowledge the support of ANR-15-CE40-002001 grant LSD. C.E. is supported by the Brasilian National Council for Scientific and Technological Development $(\mathrm{CNPq})$. Part of this work was done during the author's stay at the Institut Henri Poincare - Centre Emile Borel during the trimester « Stochastic Dynamics Out of Equilibrium $»$. The author thanks this institution for its support.

[1] M. Marchetti, J. Joanny, S. Ramaswamy, T. Liverpool, J. Prost, M. Rao, and R. A. Simha, Reviews of Modern Physics 85, 1143 (2013).
[2] P. Romanczuk, M. Bär, W. Ebeling, B. Lindner, and L. Schimansky-Geier, The European Physical Journal Special Topics 202, 1 (2012).

[3] T. Vicsek and A. Zafeiris, Physics Reports 517, 71 (2012).

[4] C. Bechinger, R. Di Leonardo, H. Löwen, C. Reichhardt, G. Volpe, and G. Volpe, Reviews of Modern Physics 88, 045006 (2016).

[5] T. Vicsek, A. Czirók, E. Ben-Jacob, I. Cohen, and O. Shochet, Physical review letters 75, 1226 (1995).

[6] J. Toner and Y. Tu, Physical Review Letters 75, 4326 (1995).

[7] V. Schaller, C. Weber, C. Semmrich, E. Frey, and A. R. Bausch, Nature 467, 73 (2010).

[8] A. Bricard, J.-B. Caussin, N. Desreumaux, O. Dauchot, and D. Bartolo, Nature 503, 95 (2013).

[9] H. H. Wensink, J. Dunkel, S. Heidenreich, K. Drescher, R. E. Goldstein, H. Löwen, and J. M. Yeomans, Proceedings of the National Academy of Sciences 109, 14308 (2012).

[10] S. Ngo, A. Peshkov, I. S. Aranson, E. Bertin, F. Ginelli, and H. Chaté, Physical review letters 113, 038302 (2014).

[11] M. E. Cates and J. Tailleur, Annual Review of Condensed Matter Physics 6, 219 (2015).

[12] I. Theurkauff, C. Cottin-Bizonne, J. Palacci, C. Ybert, and L. Bocquet, Physical review letters 108, 268303 (2012).

[13] J. Palacci, S. Sacanna, A. P. Steinberg, D. J. Pine, and P. M. Chaikin, Science 339, 936 (2013).

[14] E. Fodor, C. Nardini, M. E. Cates, J. Tailleur, P. Visco, and F. van Wijland, Physical Review Letters 117, 038103 (2016).

[15] J. Tailleur and M. Cates, Physical review letters 100, 218103 (2008).

[16] J. Tailleur and M. Cates, EPL (Europhysics Letters) 86, 60002 (2009).

[17] G. Grégoire and H. Chaté, Physical review letters 92, 025702 (2004).

[18] B. Derrida, Journal of Statistical Mechanics: Theory and Experiment 2007, P07023 (2007).

[19] E. Bertin, M. Droz, and G. Grégoire, Physical Review E 74, 022101 (2006).

[20] A. Ahmadi, M. C. Marchetti, and T. B. Liverpool, Physical Review E 74, 061913 (2006).

[21] E. Bertin, M. Droz, and G. Grégoire, Journal of Physics A: Mathematical and Theoretical 42, 445001 (2009).

[22] A. Baskaran and M. C. Marchetti, Proceedings of the National Academy of Sciences 106, 15567 (2009).

[23] A. Baskaran and M. C. Marchetti, Journal of Statistical Mechanics: Theory and Experiment 2010, P04019 (2010).

[24] T. Ihle, Physical Review E 83, 030901 (2011).

[25] A. Peshkov, I. S. Aranson, E. Bertin, H. Chaté, and F. Ginelli, Physical review letters 109, 268701 (2012).

[26] F. Thüroff, C. A. Weber, and E. Frey, Physical review letters 111, 190601 (2013).

[27] A. Solon and J. Tailleur, Physical review letters 111, 078101 (2013).

[28] E. Bertin, H. Chaté, F. Ginelli, S. Mishra, A. Peshkov, and S. Ramaswamy, New Journal of Physics 15, 085032 (2013).

[29] T. Ihle, European Physical Journal-Special Topics 223, 1293 (2014).

[30] E. Bertin, A. Baskaran, H. Chaté, and M. C. Marchetti, 
Physical Review E 92, 042141 (2015).

[31] A. Manacorda and A. Puglisi, Physical review letters 119, 208003 (2017).

[32] F. Bolley, J. A. Cañizo, and J. A. Carrillo, Applied Mathematics Letters 25, 339 (2012).

[33] P. Degond and S. Motsch, Mathematical Models and Methods in Applied Sciences 18, 1193 (2008).

[34] A. Czirók, H. E. Stanley, and T. Vicsek, Journal of Physics A: Mathematical and General 30, 1375 (1997).

[35] O. O'Loan and M. Evans, Journal of Physics A: Mathematical and General 32, L99 (1999).

[36] F. Peruani, T. Klauss, A. Deutsch, and A. Voss-Boehme, Physical Review Letters 106, 128101 (2011).

[37] A. Thompson, J. Tailleur, M. Cates, and R. Blythe, Journal of Statistical Mechanics: Theory and Experiment 2011, P02029 (2011).

[38] R. Soto and R. Golestanian, Physical Review E 89, 012706 (2014).

[39] K. R. Pilkiewicz and J. D. Eaves, Physical Review E 89, 012718 (2014).

[40] A. De Masi, P. Ferrari, and J. Lebowitz, Physical review letters 55, 1947 (1985).

[41] G. Jona-Lasinio, C. Landim, and M. Vares, Probability theory and related fields $\mathbf{9 7}, 339$ (1993).

[42] T. Bodineau and M. Lagouge, Journal of Statistical Physics 139, 201 (2010).

[43] C. Erignoux, arXiv preprint arXiv:1608.04937 (2016).

[44] Y. Fily and M. C. Marchetti, Physical review letters 108, 235702 (2012).

[45] A. P. Solon, H. Chaté, and J. Tailleur, Physical review letters 114, 068101 (2015).

[46] A. P. Solon, J. Stenhammar, M. E. Cates, Y. Kafri, and J. Tailleur, arXiv preprint arXiv:1609.03483 (2016).

[47] L. Bertini, A. De Sole, D. Gabrielli, G. Jona-Lasinio, and C. Landim, Journal of Statistical Physics 107, 635 (2002).

[48] J. Tailleur, J. Kurchan, and V. Lecomte, Journal of Physics A: Mathematical and Theoretical 41, 505001 (2008).

[49] J.-B. Caussin, A. Solon, A. Peshkov, H. Chaté, T. Dauxois, J. Tailleur, V. Vitelli, and D. Bartolo, Physical review letters 112, 148102 (2014).

[50] A. P. Solon, J.-B. Caussin, D. Bartolo, H. Chaté, and J. Tailleur, Physical Review E 92, 062111 (2015). 